\title{
Vidensque diabolus amorem tantum
}

\section{Der Teufel in Inzesterzählungen des Mittelalters}

Wenn die Literatur des Mittelalters von Inzest erzählt, hat nicht selten der Teufel seine Finger im Spiel. Seine Rolle ist unscheinbar, aber entscheidend. Er tritt im Moment anstößigen Begehrens auf und verschwindet mit dem vollzogenen oder verhinderten Inzest wieder aus der Erzählung. Der Teufel ist eine eigenständige Figur, zugleich ist seine Präsenz im Text vom Figurenverhalten abhängig. Einerseits gehört er einem allgemeinen Bösen an, das in der erzählten Welt unabhängig von den Figuren vorausgesetzt wird. Andererseits ist sein Erscheinen eng mit der verwerflichen Intention der Figuren verknüpft. ${ }^{1}$ Er reagiert, so könnte man dieses punktuelle Auftauchen verstehen, auf das Sündhafte, was sich in und zwischen den Figuren anbahnt. Der Teufel führt damit eine figürliche Schattenexistenz, die nicht selbstständig handelt, sondern Hinweise auf die prekäre Stelle innerhalb der Handlungs- und Figurenkonstellation gibt. Damit kommt ihm primär eine narrative Kommentarfunktion zu.

Die Figur des Teufels legt auch eine religiöse Perspektivierung des Inzestgeschehens nahe. Tatsächlich brauchen mittelalterliche Erzählungen die Inzestsünde als denkbar schwere Herausforderung an das Seelenheil, um so den Zusammenhang von Sünde und Buße mit dem höchsten Einsatz durchzuspielen. Doch das Inzestmotiv verbindet die Heilsfrage nicht zufällig mit den genealogischen Wirren von Herrscherfamilien. Die Texte betonen mal die politischgenealogische, mal die religiös-moralische Dimension stärker, doch der Inzest funktioniert immer als Scharnier zwischen den Diskursen. Inzest stört zugleich Herrschafts- und Heilsordnung. Er bedeutet einen doppelten Tiefpunkt, aus dem ein Weg gefunden werden muss, aus der dynastischen Enge des Familienverbundes und der moralischen Schwäche des Einzelnen. Der Teufel weist

1 Er könnte mit Paul Ric๕eUR als Grenzfigur beschrieben werden: „In die Perspektive des Sündenbekenntnisses und der sie erhellenden Symbolik eingeholt, ist die Gestalt des Bösen immer nur eine Grenzfigur, die jenes Böse bezeichnet, das ich fortsetze, wenn ich, auch ich, es beginne und in die Welt bringe; das Immerschon-da des Bösen ist die andere Sicht jenes Bösen, für das ich gleichwohl verantwortlich bin.“ RIC๕Un, Paul: Symbolik des Bösen. Phänomenologie der Schuld. Bd. 2 [Paris 1960], Freiburg/Breisgau u. a. ${ }^{2} 1988$, S. 296.

Sarina Tschachtli, Universität Heidelberg, Germanistisches Seminar, Hauptstr. 207-209, 69117 Heidelberg, sarina.tschachtli@gs.uni-heidelberg.de

Ә Open Access. ( 2021 Sarina Tschachtli, publiziert von De Gruyter. (cc) BY-NC-ND Dieses Werk ist lizensiert unter einer Creative Commons Namensnennung - Nicht-kommerziell - Keine Bearbeitung 4.0 International Lizenz. 
damit immer eine zweifache Einfallstelle der Sünde aus: Er verdeutlicht die sittliche Schwäche einzelner Figuren, doch sein Auftreten markiert auch die zu enge Vertrautheit als Schwachstelle in der Familienkonstellation. Eben das sollen im Folgenden vier Beispiele verdeutlichen.

\section{Amorem tantum (Marienmirakel)}

Die dreizehnte Erzählung der Gesta Romanorum, ein Marienmirakel, berichtet von einer schwierigen Beichte. ${ }^{2}$ Die Vergehen, die so unaussprechlich scheinen, sind die des Inzests und des Kindsmordes. Nachdem ein nicht weiter benannter König gestorben ist, teilt seine Frau das Bett mit ihrem Sohn, was wie folgt erzählt wird:

Cum autem sepulture traditus fuisset, regina per se in castro quodam vixit, habens secum filium suum, dilexitque in tantum puerum, quod ejus presencia carere non posset. Ambo simul continue jacebant, donec puer XVIII annos in etate complevisset. Vidensque diabolus amorem tantum inter matrem et puerum ejus eos ad opus nefarium sollicitavit, intantum quod filius matrem cognovit. Regina vero statim concepit. ${ }^{3}$

Als der König beerdigt worden war, lebte die Königin für sich allein auf einer Burg. Sie hatte ihren Sohn bei sich und liebte ihn so sehr, daß sie auf seine Gegenwart nicht verzichten konnte. Beide schliefen immer zusammen, bis der Junge achtzehn Jahre alt geworden war. Da sah der Teufel die große Liebe zwischen Mutter und Sohn und stachelte sie zu ruchlosem Tun an, und so geschah es, daß der Sohn mit seiner eigenen Mutter geschlechtlich verkehrte. Die Königin wurde sofort schwanger. ${ }^{4}$

Dieser Handlungsablauf ist bei aller Knappheit in mehrfacher Hinsicht typisch für mittelalterliche Inzesterzählungen. Ausgangspunkt der Erzählung ist der Tod eines legitimen Partners. Das Kind, so scheint es, kompensiert dieses Fehlen. Die Kompensation ist zunächst emotional (dilexitque in tantum puerum). Vor dem Inzest wird eine ungeschlechtliche familiäre Nähe vorausgesetzt, die den Inzest naheliegend erscheinen lässt: Der Sohn ist der Mutter nicht nur emotional der unmittelbar nächste Mann, sondern auch standesmäßig und räumlich (habens secum filium suum). Der Inzest wird als Umschlagen von einer legitimen in eine illegitime Nähe ebenso wie von einer emotionalen in eine se-

2 Das sogenannte Marienmirakel ist in verschiedenen lateinischen und volkssprachlichen Fassungen überliefert. Vgl. dazu KÜSTERS, Urban: Marken der Gewissheit. Urkundlichkeit und Zeichenwahrnehmung in mittelalterlicher Literatur, Düsseldorf 2012, S. $275 f$.

3 Gesta Romanorum. Hrsg. von Hermann Oesterley, Hildesheim 1963 [Berlin 1872], S. 291.

4 Gesta Romanorum. Hrsg. von Rainer NicKeL, Lateinisch/Deutsch, Stuttgart 1991, S. 25. 
xuelle Beziehung erzählt. Wenngleich der Teufel als Auslöser des Inzests auftritt (eos ad opus nefarium sollicitavit), so wird dieser doch schlüssig aus der innerfamiliären Dynamik hergeleitet: vom Fehlen des Partners, über die familiäre Nähe, die im Teilen des Bettes den deutlichsten Ausdruck findet (Ambo simul continue jacebant).

Zugleich scheint der Zeitpunkt dieses Umschlagens nicht beliebig, sondern an das Älterwerden des Sohnes gebunden (donec puer XVIII annos in etate complevisset). Das Umschlagen von einer emotionalen in eine sexuelle Beziehung mag also vom Teufel ausgelöst werden, die Möglichkeit ist aber in der Ausgangslage der familiären Nähe und des älter werdenden Sohnes bereits angelegt. Entsprechend knapp ist die Intervention des Teufels. Er sieht das Liebesverhältnis (amorem tantum) und nimmt Einfluss darauf, indem er zu einer geschlechtlichen Handlung anstiftet. Dem Teufel kommt damit nur wenig eigene Präsenz im Text zu. Die Einflussnahme wird nicht weiter ausgestaltet oder auserzählt, vielmehr markiert der Teufel die Einfallstelle des Geschlechtlichen in die verwandtschaftliche Beziehung.

Der Inzest führt deshalb auch unweigerlich zur Zeugung - die Mutter wird sofort (statim) schwanger. Die Zeugung wiederum ist eine narrative Notwendigkeit, um das zunächst heimliche inzestuöse Verhältnis in eine Krise zu führen, weil es durch das Kind offenbar wird. Mit der zentralen Funktion der Zeugung im Text geht auch eine für die mittelalterlichen Inzesterzählungen typische Fokusverschiebung von der Vater- auf die Mutterfigur einher. Der König stirbt ohne weiteren Hinweis auf seine Person, sein Sohn sucht die Weite. Mit der Schwangerschaft ist die Mutter Trägerin des sündhaften Zeichens - des Kindes. Nach der Niederkunft tötet die Mutter das Kind, doch vier Blutstropfen fallen auf ihre Hand und können nicht mehr abgewaschen werden. Mit der Tötung des Kindes verdoppelt sich die Sünde, das Zeichen der Sünde wiederum vervierfacht sich in den Blutstropfen und geht auf die Mutter über. Es ist in beiden Teilen der Erzählung die Mutter, welche die Markierung der Sünde trägt. Die Königin verhält sich nun fromm und mildtätig, sie beichtet ihre Sünden, nicht aber den Inzest und die Tötung des Kindes. Durch eine Offenbarung der Jungfrau Maria an den Beichtvater bringt dieser die Königin schließlich doch dazu, den Inzest zu gestehen. Damit wird gerade der Inzest für heilsdidaktische Zwecke genutzt, doch wird in der Auflösung nicht die Sünde problematisiert, sondern die vermeintliche Unaussprechlichkeit derselben. Im Beichtimperativ der Erzählung wird also gerade die tabuisierte sexuelle Handlung für die entscheidende Einsicht gebraucht: In der Beichte ist nicht einmal Inzest tabu. 


\section{Bey einander fchlaffen (Albanus)}

Auch im zweiten Beispiel wird Inzest als Extremform der Sünde funktionalisiert. Die Albanuslegende ist im zwölften Jahrhundert entstanden und liegt in verschiedenen lateinischen Vers- und Prosafassungen vor. ${ }^{5}$ Albrecht von Eyb überträgt im fünfzehnten Jahrhundert die lateinische Prosafassung $C$ ins Deutsche (auf dieselbe Fassung geht auch die Albanuslegende zurück, die teilweise in den Gesta Romanorum mitüberliefert wird). Die Erzählung scheint sich zunächst wie die erste zu entwickeln, mit umgekehrten Geschlechterrollen: Als die fraw mit tod abgangen was, da ward der kůnig fein augen werffen in fein leipliche tochter, fie lieben vnd befchlaffen vnd aus ir geperen ein fun, den der kůnig wolt getót haben, die groffen fůnde damit zuuerdecken (S. 91; „Als die Frau gestorben war, da begann der König seine Augen auf seine leibliche Tochter zu richten, sie zu lieben und mit ihr zu schlafen. Sie gebar ihm einen Sohn, den der König töten lassen wollte, um die große Sünde zu verdecken“). ${ }^{6}$ Wieder ist der Tod des legitimen Partners Ausgangspunkt der Erzählung. Die Tochter ist dem Vater die unmittelbar nächste Frau: standesmäßig, emotional und räumlich. Zudem ist sie in unmittelbarer Sichtweite (da ward der kůnig fein augen werffen). Inzest ist auch hier wortwörtlich naheliegend. Wieder führt der Inzest umgehend zur Zeugung, doch anders als im Marienmirakel ist das Kind nicht nur Zeichen der Sünde, sondern wird zum Protagonisten der Erzählung. Die Tochter und Mutter lässt das Kind, versehen mit einem teuren Mantel und reichen Gaben, entgegen der väterlichen Anweisung außer Landes bringen. Das Kind kommt an den Königshof in Ungarn und wird dort auf den Namen Albanus getauft. Es wird vom König als Sohn angenommen und herrschaftlich erzogen und tritt schließlich dessen Nachfolge an. In seinem Herkunftsland hingegen hört der Vater von Albanus und gibt ihm, ohne seine Herkunft zu erahnen, seine Tochter zur Frau. Die beiden werden vermählt, und niemand ahnt, das die neů prawt was ein muter vnd fchwester des preůtigams vnd kůnigs Albani (S. 92; „dass die neue Braut Mutter und Schwester des Bräutigams, König Albanus, war“). Erst als Albanus seiner Frau seine Herkunft eröffnet, erkennt sie, dass sie seine Mutter ist.

Auf diesem Erkenntnismoment liegt der gestalterische Fokus der Prosafassung $\mathrm{C}$ und von Eybs Bearbeitung. Alle anderen Handlungszusammenhänge sind stark gerafft erzählt. In den gegenseitigen Erklärungen und Schreckensbekundungen

5 Zum Verhältnis der deutschen Fassungen zur lateinischen Überlieferung vgl. MoRvaY, Karin: Die Albanuslegende, München 1977 (Medium Aevum. Philologische Studien 32).

6 Hier und im Folgenden zitiert nach: Deutsche Schriften des Albrecht von Eyb. Hrsg. und eingeleitet von Max HERRMAnN. Band 1, Berlin 1890 (Schriften zur Germanischen Philologie 4). 
hingegen wird die komplexe Familienkonstellation mehrfach thematisiert. So versteht Albanus zunächst den Schrecken seiner Frau falsch; er meint, sie lehne ihn ab, weil sie nun wisse, dass er ein Findelkind sei. Sie aber versichert ihn einer der familiären Nähe gemäßen Zuneigung - du bift mir nit zuuerfchmehen, fo hab ich dich billich lieb: Wann du bift mir auß meinem leiplichen vater ein bruder vnd ein fun vnd aus bruder vnd fun ein haußwirt (S. 94; ,ich verachte dich nicht, denn ich habe dich gebührend lieb: Du bist mir durch meinen leiblichen Vater ein Bruder und ein Sohn, und aus Bruder und Sohn ein Ehemann geworden“). Auch die Erkenntnis des Vaters, im Schwiegersohn den Sohn wiederzufinden, wird als ein Moment von Schrecken und Vertrautheit eingeführt: die tochter fprach: „Es ift nit, das wir vnns vor im follen forchten vnd scheůhen: er ift der, den du, vater, auß mir, deiner tochter, haft geboren [. . . ““ (S. 95f.; „die Tochter sprach: ,Wir sollen uns vor ihm nicht fürchten und scheuen: Er ist der, der dir, Vater, durch mich, deine Tochter, geboren wurde [. . .]“"). Hier überblenden sich also im Inzest in eigentümlicher Weise eine positiv konnotierte familiäre Verbundenheit mit der Illegitimität der geschlechtlichen Beziehungen. Der Text zieht seine erzählerische Drastik aus der Komplexität der Familienkonstellation und wendet diese in einen Schrecken über die eigene Sündhaftigkeit. Zugleich inszeniert die Entdeckung auch eine Vervielfachung der familiären Nähe und Intimität.

Dass legitime Emotion und illegitimes Begehren so nahe beieinander liegen, prägt auch den weiteren Verlauf der Inzestlegende. Albanus und seine Mutter beichten die Sünden schließlich zusammen mit dem Vater einem Bischof und einem Einsiedler, werden zu sieben Jahren Buße auf Wanderschaft aufgefordert, was sie willig antreten. Die Beichte und die gemeinsame Bußwanderung legen nahe, dass die geschlechtlichen Beziehungen aus den familiären Relationen getilgt werden können, doch zeigt sich auch diese Konstellation als prekär. Auf dem Rückweg verirren sie sich im Wald und schlagen dort ihr Nachtlager auf. Albanus bereitet den Eltern ein Bett und steigt selber auf einen Baum. Hier nun hat der Teufel seinen Auftritt:

Als nun vater vnd tochter bey einander fchlaffen follten, do wachet der po̊fe geift vnd veind der menfchen, bewegt vnd ůberwand vater vnd tochter, das fie wider von neůem einfielen in die miffetat der vnlauterkeit vnd sich miteinander vermifchten.

Als nun Vater und Tochter beieinander schlafen sollten, da erwachte der böse Geist und Feind der Menschen, bewegte und überwand Vater und Tochter, so dass sie erneut die Missetat der Unlauterkeit begingen und sich vereinten.

Die körperliche Nähe des Schlafarrangements scheint den Teufel hervorzurufen: Vater und Tochter legen sich zueinander, doch sie schlafen nicht, zudem erwacht der Teufel. Nicht nur die Nähe im Waldbett ist verhängnisvoll, sondern auch die Vertrautheit aus der Zeit des früheren Inzests - die beiden finden wider von 
neůem zueinander. Die Einflussnahme des Teufels wird dabei körperlich und kämpferisch beschrieben (bewegt vnd überwand), obwohl sich ja gerade kein kämpferisches, sondern ein sexuelles Ineinander abspielt. Das Eingreifen des Teufels findet also auf einer anderen Ebene der Handlung statt: Die Szene überblendet das eigentliche, körperliche Geschehen mit dessen Bedeutung für das Seelenheil der Figuren, was als Widerstreit mit dem Teufel verbildlicht wird. Die körperliche Vereinigung ist zugleich ein verlorener Kampf mit dem Bösen.

Die Beschreibung des Teufels als po̊fe[r] geift legt nahe, dass ihm nicht die gleiche körperliche Präsenz zukommt wie den Figuren. Dennoch wird auf beiden Ebenen der Beschreibung, in der unmoralischen Handlung und im Widerstreit mit dem Teufel, körperliche Nähe aufgerufen. Auch die Begrifflichkeit, die Eyb für den Geschlechtsakt verwendet, zielt auf eine Extremform der Nähe: auf Vermischung. (In den Gesta Romanorum heißt es ähnlich: pater filie recidive flagicioseque commiscebatur. ${ }^{7}$ ) Diese Vermischung ist ganz körperlich als geschlechtliche Vereinigung lesbar, lässt sich aber auch auf die vom Inzest vermischten Familienrelationen beziehen. ${ }^{8}$ Das Eingreifen des Teufels markiert also eine mehrfache Einfallstelle: Das geschlechtliche Begehren fällt in die verwandtschaftlich-emotionalen Beziehungen ein, die Sünde in die Bußbemühungen, und die relationale Verunklarung in die zuvor distinkten familiären Kategorien. Die narrative Funktion des Teufels an dieser Stelle liegt jedoch nicht einfach darin, den Inzest auszulösen - dazu war er auch beim ersten Inzest nicht nötig. Er markiert vielmehr die Einfallstelle und verdeutlicht damit, dass es hier einen kategorialen Wechsel zwischen der legitimen und der illegitimen Relation gibt, der aber in der Situation in prekärer Weise als kontinuierlicher Übergang zwischen familiärer Nähe und Geschlechtlichkeit erscheint. Der Teufel weist einen Kippmoment im Handeln der Figuren aus, gerade weil dieses Handeln in unguter Weise naheliegend sein könnte.

7 Gesta Romanorum, S. 645 (Anm. 3); „der Vater vereinte sich erneut schändlich mit der Tochter."

8 In einem weiteren Schritt lässt sich die Vermischung auch als Ent-Differenzierung im Sinne von Peter StroHSCHNEIDERs ,Inzest-Heiligkeit" verstehen. STROHSCHNEIDER beschreibt Inzest als kulturelle Krise, indem er von der genealogischen Ordnung auf eine Ordnung allgemein schließt: „Die Erzählung verwendet das Verbrechen [i. e. den Inzest] als Problematisierungsfigur und damit kommunikativ als Mechanismus der Stabilisierung von genealogischer Ordnung, hier also Ordnung allgemein. Das Verbrechen, der Inzest, erscheint theologisch als Todsünde, strukturell aber als ,Krise der Unterschiede‘, als Krise von Kultur überhaupt, die auf den vom Gesetz begründeten Unterschieden aufbaut.“ STROHSCHNEIDER, Peter: Inzest-Heiligkeit. Krise und Aufhebung der Unterschiede in Hartmanns ,Gregorius‘. In: Geistliches in weltlicher und Weltliches in geistlicher Literatur des Mittelalters. Hrsg. von Christoph HuBER/Burghart WACHINGER/Hans-Joachim ZIEGELER, Tübingen 2000, S. 105-133, hier S. 118. 
Der Teufel tritt damit auch als Markierung einer unguten Nähe auf. Er greift in die Situation ein und beeinflusst die Figuren, ist dabei aber auf die bereits gegebene Nähe in der Schlafsituation angewiesen. Er scheint also gerade durch die allzu nahe Familienkonstellation hervorgerufen. Der Teufel nutzt eine sittliche Schwachstelle in der Familie, er markiert sie damit aber auch als solche. So gesehen scheint es auch nicht plausibel, dass der Teufel hier - eine mögliche Argumentation - die Figuren entlastet. Der Teufel agiert nicht als selbstständige Figur des Bösen, die dieses Böse außerhalb der Figuren verortet, wie es das Verständnis des Teufels als Verführer nahelegt. Vielmehr erscheint er als personifizierter Indikator einer in der sozialen Konstellation bereits angelegten Gefahr, die er nicht schafft, sondern als solche ausweist.

Albanus sieht den zweiten Inzest, steigt vom Baum und erschlägt seine Eltern. Diese Tötung ist ein Spezifikum der Albanuslegende, andere Erzählungen finden für inzestuöse Eltern Möglichkeiten der Vergebung. Dass Albanus seine Eltern tötet, ist als gezielte Auslöschung seiner eigenen Herkunft lesbar. Er beendet den Inzest, dessen Produkt er selber ist. Die heimliche Sünde wird dabei zum zweiten Mal durch Albanus sichtbar gemacht. Zuvor bezeugt das Kind den Inzest, dann wird es selbst sein Zeuge. Doch so außergewöhnlich Albanus' Verstrickung in die Schuld seiner Familie erscheint, sie ist - als Geburt in eine sündige Welt und in familiäre Bindungen - auch eine existenzielle Grundbedingung. Das Erzählen vom Inzest wird in der mittelalterlichen Legendarik also auch eine Auseinandersetzung mit der Sünde, die einem schon in die Wiege gelegt ist: der Erbsünde.

Die Drastik, mit der sich Albanus gegen seine eigene Herkunft wendet, führt tiefer in die Sünde, doch ist die Negation der eigenen, vorbelasteten Herkunft auch die Voraussetzung für einen Weg aus der familiären Sündenfalle. ${ }^{9}$ Die Tötung der Eltern ist nach weiteren sieben Jahren Wanderschaft gebüßt und der Weg zu Albanus' Heiligung geebnet. Es ist letztlich nicht der Vollzug des Inzests, der die Erzählung nachhaltig beschäftigt, sondern der weite Weg aus diesen Fallstricken der Familie. Der Inzest führt zum Ausschluss aus der Gesellschaft, was sich auch in der Weite der erzählten Welt zeigt. Nicht nur wird das Kind der inzestuösen Verbindung ausgesetzt und anderenorts erzogen, auch die reuigen Sünder suchen nach der Einsicht in die eigene Sündhaftigkeit in Bußbemühungen die Weite. Der Inzest wird so zum wortwörtlichen Beweggrund der Erzählung, und die Sünde wird zum Ausgangspunkt des Heils.

9 „Endpunkt ist eine Konstellation, in der familiale Bindungen aufgehoben, genealogische Linien abgeschnitten sind. Dabei spielt Gewalt eine entscheidende Rolle. Sie ist Ursache wie Effekt der genealogischen Krise. Sie ist zugleich Ausdruck des Systems und Bedingung seiner Veränderung.“ KIENING, Christian: Unheilige Familien. Sinnmuster mittelalterlichen Erzählens, Würzburg 2009 (Philologie der Kultur 1), S. 72. 


\section{Der triuwen alze vil (Gregorius)}

Auch bei Hartmanns von Aue Gregorius ist der Inzest ein Moment der Abscheu und Beweggrund der schließlichen Erhöhung. ${ }^{10}$ Hier kommt es zum Inzest zwischen Zwillingen. Hartmann versprachlicht das erstaunlich lakonisch: dâ was der triuwen alze vil (V. 395; „Das war wirklich zu viel der treuen Sorge“). ${ }^{11}$ Der Begriff der triuwe verweist hier auf die höfische Exemplarität der zwei Geschwister und ihre gegenseitige Verbundenheit - alze vil hingegen markiert eine Überschreitung, bei der die angemessene Verbundenheit in eine ungehörige Verbindung kippt. Ist also die Steigerung von triuwe ins Ungeheuerliche bei Gregorius' Zeugung nur ein graduelles Zuviel, wie Hartmanns Formulierung nahelegt? Doch hat auch hier der Teufel seine Finger im Liebesspiel. Das Eingreifen des Teufels suggeriert, dass hier ein kategorialer Wechsel vor sich geht - und das ist vielleicht die entscheidende Funktion der Figur.

Die Rolle des Teufels wird hier ausführlicher beschrieben als in den ersten beiden Beispielen. Er nimmt die bestehende Nähe und Eintracht zwischen den Geschwistern (und insbesondere die große Liebe des Bruders zur Schwester) wahr (V. 303f.). Auch hier wird die nächtliche Nähe erwähnt: ir bette stuonden alsô nâ / daz si sich mohten undersehen. (V. 294f.; „Ihre Betten standen so nah beieinander, daß sie sich sehen konnten.“) Der Teufel nutzt eine Empfindung, die sich in der liebevollen Fürsorge des Bruders für seine Schwester schon abzeichnet, die hier aber in etwas anderes verkêret ${ }^{12}$ wird: an sîner swester minne / so riet er [i. e. der Teufel] im ze verre / unz daz der juncherre / verkêrte sîne triuwe guot / ûf einen falschen muot. (V. 318-322; „Liebesverlangen nach seiner Schwester flüsterte er ihm so lange ein, bis der junge Herr seine aufrichtige Liebe in

10 Zum Verhältnis der Albanus- und Gregorius-Stoffe vgl. KNAPP, Fritz Peter: legenda aut non legenda. Erzählstrukturen und Legitimationsstrategien in ,falschen` Legenden des Mittelalters: Judas - Gregorius - Albanus. In: Germanisch-Romanische Monatsschrift, N. F. 53 (2003), S. 133-154.

11 Hier und im Folgenden zitiert nach: Hartmann von Aue: Gregorius, Der Arme Heinrich, Iwein. Text und Kommentar. Hrsg. und übersetzt von Volker MERTENS, Frankfurt a. M. 2004 (Bibliothek deutscher Klassiker. Bibliothek des Mittelalters 6).

12 Hallich nimmt u. a. diese Stelle als Beleg, dass der Inzest „durch das Wirken des Teufels [begründet]“ werde, stellt aber auch fest, dass die syntaktischen Relationen das Agens dem Bruder zuschreiben (der juncherre / verkêrte sîne triuwe guot). HaLlicH, Oliver: Poetologisches, Theologisches. Studien zum ,Gregorius‘ Hartmanns von Aue, Frankfurt a. M. u. a. 1995, S. 62f. 
falsches Begehren verkehrte.“). Trotz dieser deutlichen Beschreibung seiner Einflussnahme wird expliziert, dass der Teufel nur einer von vier Faktoren ist:

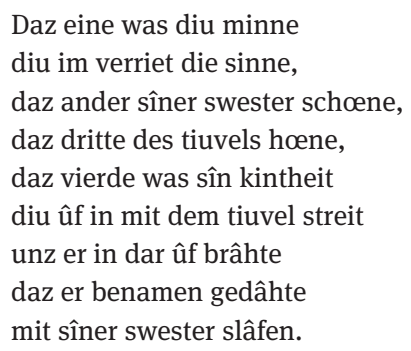

(V. 323-331)

Das eine war das Liebesverlangen, das ihm den Kopf verdrehte, das zweite die Schönheit seiner Schwester, das dritte des Teufels Falschheit, das vierte seine kindliche Unerfahrenheit, die sich im Bund mit dem Teufel gegen ihn wandte, bis er ihn dazu brachte, daß er tatsächlich darauf aus war, mit seiner Schwester zu schlafen.

Der erste Faktor ist also die minne, die auch unabhängig von der teuflischen Eingabe den Inzest mitverschuldet, der zweite die Schönheit der Schwester. Erst der dritte Faktor ist des tiuvels hœene (V. 326). Des tiuvel[s] streit (V. 326) wiederum verbindet sich mit dem vierten Faktor: des Bruders kintheit (V. 327). Diese Aufzählung macht deutlich, dass der Teufel nicht monokausaler Auslöser des Inzests ist, sondern sich mit der gegebenen Ausgangslage verbindet: mit der Zuneigung zwischen den Geschwistern, ihrer adligen Schönheit und bisher unschuldigen Kindlichkeit. Eben diese situativen Faktoren sind aber nicht negativ konnotiert oder sündhaft - im Gegenteil. Erst in Verbindung mit dem Teufel wird ihr Gefahrenpotential verdeutlicht und ausgespielt. ${ }^{13}$

Dieses Teufelsverhalten wird als listig qualifiziert. Der Erzähler warnt: wâfen, herre, wâfen / über des hellehundes list / daz er uns sô geværic ist! (V. 332-334;

13 Vgl. dazu Albrecht HAUSMAnN: „Erst in ihrem Zusammenspiel und durch den Einfluss des Teufels wirken sich diese Faktoren negativ aus; für sich genommen erscheinen minne und schoene, potentiell aber auch kintheit - im Sinne von ,Unschuld, Naivität - durchaus positiv. Der Teufel pervertiert diese Faktoren zur sexuellen Lust (daz er benamen gedâhte / mit sîner swester slâfen, Gregorius, V. 330 f.).“ HAUSMANN, Albrecht: Gott als Funktion erzählter Kontingenz. Zum Phänomen der ,Wiederholung، in Hartmanns von Aue Gregorius. In: Kein Zufall. Konzeptionen von Kontingenz in der mittelalterlichen Literatur. Hrsg. von Cornelia HERBERICHS/Susanne REICHLIN, Göttingen 2010, S. 79-109, hier S. 92. Dazu auch Ingrid KaStEN: „Gegenüber dieser psychologischen Motivation tritt die Bedeutung des Teufels an dem Geschehen merklich in den Hintergrund.“ KASTEN, Ingrid: Schwester, Geliebte, Mutter, Herrscherin. Die weibliche Hauptfigur in Hartmanns ,Gregorius‘. In: PBB 115 (1993), S. 400-420, hier S. 406. 
„Weh, Herr Gott, o weh über diese Künste des Höllenhundes, daß er uns so in Gefahr bringt!“) Doch es ist nicht des Teufels Tat, die hier erzählt wird. Er greift nicht aktiv in das Geschehen ein, sondern wirkt mit Rat. Die missetât bleibt dem Bruder zugeschrieben: Dô er [i. e. der Bruder] durch des tiuvels rât / dise grôze missetât / sich ze tuonne bewac (V. 339-341; „Als er aufgrund der Einflüsterungen des Teufels diese große Sünde zu begehen sich vornahm“). ${ }^{14}$ Die Schwester ist im Vergleich dezidiert passiv, der Wille des Teufels wird an ir vollbracht: nû begap si der tiuvel nie / unz sîn wille an ir ergie. (V. 351f.; „Jetzt ließ sie der Teufel nicht los, bis er seinen Willen an ihr vollbrachte.“) Dass der Teufel die Schwester nicht mehr loslässt, beschreibt eine Handlung des Teufels, ${ }^{15}$ dennoch ist es zweifellos der Bruder, der die Schwester überwältigt (also auch festhält). Der Wille des Teufels wird durch den Bruder vollzogen, und er ist nicht zufällig deckungsgleich mit dem Wollen des Bruders.

Einmal begonnen, finden dann beide Geschwister Gefallen am neuen Spiel: der tiuvelschünde luoder / begunde si mêre schünden, / daz in mit den sünden / lieben begunde. (V. 400-403; „Die teuflische Verlockung des Triebes reizte sie um so heftiger, so dass sie bei dieser Sünde Lust und Freude hatten.“) Ist die Tat einmal vollzogen, braucht es den Teufel nicht mehr als Agens, wie diese Passage zeigt; er ist nur noch adjektivisch präsent. Das Teuflische ist nun in Empfindung und Handlung - im luoder - enthalten. Der Teufel muss nicht mehr ins Geschehen eingreifen, vielmehr ist seine Wirkung bereits in den Akt der Geschwister übergegangen.

Der Text benennt schließlich die vormalige Nähe zwischen den Geschwistern als eigentlichen Grund der Verführung, genauer die Vertrautheit oder Heimlichkeit: in geschach diu geswîche / von grôzer heimelîche: / heten si der entwichen / sô wæren si unbeswichen. (V. 411-414; „Sie verfielen der Verführung, weil sie zu vertraut miteinander waren - hätten sie das vermieden, wären sie nicht betrogen worden.") Der Teufel verkörpert also auch hier das Gefahrenpotential, das in der

14 Die ,Beratung، durch den Teufel wiederholt sich vor dem zweiten Inzest, wenn die Mutter Gefallen am Sohn findet. Doch der Teufel erhält hier keinerlei szenische Präsenz, vielmehr wird er vom Erzähler formelhaft aufgerufen: ouch behagete ir der gast / baz danne ie man getæte. / daz macheten sîne ræte / der ouch vroun Êven verriet (V. 1958-1961; „und ihr gefiel der Fremde auch besser als jemals ein Mann: das war der Plan des Verführers, der auch Frau Eva verleitet hat"). Eher als eine Verführung durch den Teufel wird mit dem Verweis auf Eva die Verführbarkeit von Frauen im Allgemeinen angedeutet.

15 „Der Erzähler begründet den Inzest durch das Wirken des Teufels, den er zum eigentlich Agierenden macht, indem er sein Wirken auf die Geschwister mit größtenteils aus dem Prolog übernommenen, das Gewalthafte der Teufelseinwirkung betonenden Metaphern beschreibt.“ Hallich, Poetologisches, Theologisches, S. 62 (Anm. 12). 
Nähe der Geschwister angelegt ist. ${ }^{16}$ Er bestimmt aber nicht selbst die Handlung, sondern steigert die prekäre Handlungstendenz des Bruders, wobei ihm die Passivität der Schwester zuarbeitet. Der Teufel markiert damit den entscheidenden Kippmoment von legitimer familiärer Nähe in eine illegitime geschlechtliche Verbindung, die dann von der Schwangerschaft bezeugt wird. Der Teufel ist hier nicht Figur eines den Figuren fremden Bösen, vielmehr problematisiert sein Auftreten die Innigkeit der familiären Konstellation, die ins Verwerfliche kippen kann. Der Teufel verschuldet das Kippen nicht (allein), weist es aber narrativ als solches aus. Er verdeutlicht den Moment, in dem die geschwisterliche Zuneigung zu geschlechtlicher Liebe wird. ${ }^{17}$ Dort, wo die familiäre Nähe sich scheinbar kontinuierlich steigert, legt also gerade die Einwirkung des Teufels einen kategorialen Wechsel der Beziehung nahe. Der Teufel weist so die Einfallstelle für genealogische Unordnung in vorerst geordnete familiäre Verhältnisse aus. Gerade in diesem Moment der vermischten und verunklarten Relationen ist der Teufel ein narratives Mittel der Vereindeutigung.

\section{An minnen gar geschendet (Beaflor)}

In Mai und Beaflor ${ }^{18}$ ist es der Königsvater, der den Inzest nicht begeht, aber doch begehrt. Nachdem die Mutter der Protagonistin Beaflor gestorben ist, sieht ihr Vater sie mit anderen Augen und wird allzu vertraut mit ihr. ${ }^{19}$ Doch die unschuldige Tochter nimmt die Zärtlichkeit ganz arglos wahr und freut sich darüber: ${ }^{20}$ In der Unkenntnis seiner Intention erscheint die Vertrautheit als väterliche Zunei-

16 Daraus ergibt sich, dass der Text Verwandtschaftssysteme problematisiert, was auch STROHSCHNEIDERs Lektüre nahelegt: „Worauf es ankommt, ist allein die These, daß die totalen Katastrophen von in Verwandtschaftssystemen organisierter Herrschaft eben deswegen immer wieder - und so auch im ,Gregorius‘ - als Inzest erzählt werden, weil es dieses Gesetz ist, das Verwandtschaft allererst konstituiert.“ STROHSCHNEIDER, Inzest-Heiligkeit, S. 117 (Anm. 8).

17 Trotz dieser Vereindeutigung zeichnet Hartmann die Beziehung der Geschwister auch nach dem Inzest nicht nur negativ, sondern verleiht ihnen ,programmatisch den Status eines Minnepaares“. KASTEN, Die weibliche Hauptfigur, S. 406 (Anm. 13).

18 Hier und im Folgenden zitiert nach: Mai und Beaflor. Minneroman des 13. Jahrhunderts. Hrsg. von Christian KIENING/Katharina MERTENS FlEURY. www.ds.uzh.ch/kiening/Mai_und_Bea flor/MaiundBeaflor.pdf. Zürich 2008 (12. August 2019).

19 Swanne sei der chunnich sus vant, so gie er zu ir alzehant / vnd troute vnd chuste sie / vnd wont ir gutlichen pi. (V. 770-773; „Wenn der König sie so vorfand, ging er sogleich zu ihr, umarmte und küsste sie und war ganz freundlich zu ihr.")

20 daz nam si von im gar fur vol / vnd tet ir in ir herzen wol, / daz er sie so gerne sach. (V. 774-776; „Dies nahm sie sehr gerne von ihm an, denn es tat ihr im Herzen wohl, dass er sie so gerne sah.“) 
gung. Noch zeigt sich kein Unterschied in der Art der familiären Nähe - und in eben dem Moment wird der vil leide Sathanas (V. 784; „,wirklich bösartige Satan“) geschäftig. ${ }^{21}$ Der Teufel wird gerade in dem Moment aufgerufen, wo eine prekäre Nähe bereits etabliert ist, aber durch den naiven Blick der Tochter entschärft wird.

Doch kann der Teufel zunächst nichts ausrichten. Er versucht erst die Tochter für die Sünde zu gewinnen, erreicht aber aufgrund ihrer gottesfürchtigen Art nichts und wendet sich dem Vater zu: der tivel im di sinne / gantzlich het erblendet / vnd in an minnen gar geschendet (V. 823-825; „Der Teufel hatte ihm die Sinne ganz verblendet und ihn in der Liebe entehrt“). Hier wird nun der Einfluss des Teufels explizit gemacht: Er wirkt auf die Sinne des Königs ein. Doch auch als der Vater nach der Einwirkung des Teufels deutlicher wird und die Tochter zum minnespil (V. 878; „Liebesspiel“) auffordert, lacht sie zunächst ${ }^{22}$ - so weit scheint eine Transgression zunächst von ihrem Sinnhorizont entfernt. Doch sie realisiert, dass der Vater sich hier unangemessen verhält: ,lieber vater, wi tustu so, / was wedeutestu hie mit? / es ist wider vatersit / daz du so versuchest mich [....]' (V. 881-884; „Lieber Vater, was machst du denn? Was meinst du hiermit? Es gehört sich nicht für einen Vater, dass du mich so verführst [. . .]“). Der Übergriff des Vaters verkennt ihren Status als Tochter, zwingt Beaflor in die Position ihrer Mutter, woran sie ihn auch umgehend erinnert. Der Inzest ist ein generationaler Konflikt - er würde einen „genealogischen Kurzschluss zwischen Vater und Tochter“ ${ }^{23}$ bedeuten und die Erbfolge stören. Was der Text aber durch das zuvor ausführlich thematisierte Fehlen der Mutter noch deutlicher ausstellt, ist der emotionale Kurzschluss zwischen Mutter und Tochter. Das Fehlen der Mutter wird explizit als handlungsauslösendes Moment ausgewiesen. ${ }^{24}$

21 Der vil leide Sathanas, / der ie gevar dem menschen was, / der was vnmuzich hier vnder. (V. 784-786; „Der wirklich bösartige Satan, der dem Menschen schon immer eine Gefahr war, blieb hier nicht untätig.“)

22 bei der hant er sie do vie: / ,wir suln bedev ensampt hie / ein minnespil machen. / div tohter begunde lachen (V. 876-879; „Da nahm er sie bei der Hand: ,Wir werden jetzt hier beide zusammen ein Liebesspiel machen. 'Die Tochter fing an zu lachen“).

23 Sтоск, Markus: Herkunft und Hybridität. Biopolitics of Lineage in Mai und Beaflor. In: Hybridität und Spiel. Der europäische Liebes- und Abenteuerroman von der Antike zur Frühen Neuzeit. Hrsg. von Martin BAISCH/Jutta EmING, Berlin 2013, S. 93-111, hier S. 102.

24 V. 488-495. Das Fehlen der Mutter Beaflors wird im zweiten Teil des Romans durch eine allzu präsente (Schwieger-)Mutter kontrastiert, die eine legitime Verbindung von Beaflor mit ihrem Sohn Mai verhindern möchte. Die Frage, ob das Verhalten der Mutter Mais, analog zu Beaflors Vater, als inzestuös zu verstehen sei, beantwortet Jutta EmING mit Bezug zu den inzesttheoretischen Arbeiten von Sigmund FREUD. Sie schlägt eine begriffliche Unterscheidung zwischen dem Inzest und dem Inzestuösen vor, wobei beim Inzestuösen nicht die Frage des Figurenbegehrens im Zentrum stehe, sondern die Frage nach einer exogamen Partnerwahl, die aus dem Inzesttabu folgt, also der Wahl eines Liebesobjektes außerhalb der Familie. Erst diese exogame Partnerwahl, 
Auch hier wird also der Inzest aus einer familiären Dynamik hergeleitet, bevor der Teufel überhaupt auftritt. Der Teufel fungiert erneut als Vereindeutigung des Moments, in dem die Situation ins Illegitime kippt. Einerseits macht seine Einwirkung den Unterschied zwischen der Zärtlichkeit aus, über die die Tochter sich freut, und der sexuellen Forderung, die sie zurückweist. Zugleich wird, da der Teufel auf die Tochter keinen Einfluss nehmen kann, die Legitimität von Beaflors Position ausgewiesen, selbst wenn sie dem zuvor versprochenen Gehorsam gegenüber dem Vater zuwiderhandelt. Der Teufel tritt auch hier in dem Moment auf, in dem die distinkten familiären Relationen und die verwandtschaftlichen Positionen (der Mutter und der Tochter) durch den versuchten Inzest verunklart werden. Damit hat der Teufel erneut eine indizierende Funktion, weil er die familiäre Nähe als fragwürdig ausweist, gerade wenn sie im Blick der Figuren - der unwissenden Tochter ebenso wie des begehrenden Vaters - in prekärer Weise selbstverständlich scheinen könnte.

Bevor der Teufel seinen Einfluss auf die Figuren ausüben will, wird sein Wirken in der Welt (und der Erzählung) insgesamt thematisiert:

der [i. e. der Teufel] geschuef, daz ein wunder

vnd ein vndpilde ergie.

daz ist sein sit gewesen ie:

daz vbel zu gute

chert nach sinem mute

(V. 787-791)

Der [i. e. der Teufel] bewirkte es, daß Wundersames und Unrechtes geschah. Das war aber schon immer seine Art gewesen, daß er in seinem Ansinnen das Böse zum Guten verkehrt

so FREUDS Verständnis des Inzesttabus, ermögliche einen kulturellen Austausch zwischen Familien und sei damit konstitutiv für die Gesellschaft. Mais Mutter handle also inzestuös, nicht weil sie ihren Sohn begehrt, sondern weil sie ihre Familie gegen diesen kulturellen Austausch verschließen will. EmING, Jutta: Inzestneigung und Inzestvollzug im mittelalterlichen Liebes- und Abenteuerroman (Mai und Beaflor und Apollonius von Tyrus). In: Historische Inzestdiskurse. Hrsg. von DERS./Claudia JARZEBOWSKI/Claudia UlBRICH, Königstein/Taunus 2003, S. 21-45. Dass sich Mais Mutter dabei auf eine geläufige „,männlich“-feudale[ ] Sexual- und Eheauffassung“ beruft, zeigt KASTEN, Ingrid: Ehekonsens und Liebesheirat in Mai und Beaflor. In: Oxford German Studies 22 (1993), S. 1-20, hier S. 15. Die beiden handlungslogisch disparaten Teile werden also gerade durch eine von der Elterngeneration irritierte Erbfolge und eine Auseinandersetzung mit problematisierter Mutterschaft verbunden. Vgl. dazu: „Die Elterngeneration wendet sich gegen die Fortsetzung der Genealogie durch die Kinder und muß erst aus dem Weg geräumt werden, die ideale unproblematische Herrschaftssukzession, wie wir sie z. B. aus dem Erec kennen, gibt es nicht mehr.“ MERTENS, Volker: Herrschaft, Buße, Liebe. Modelle adliger Identitätsstiftung in ,Mai und Beaflor'. In: German Narrative Literature of the Twelfth and Thirteenth Century. Hrsg. von Volker HONEMANN u. a., Tübingen 1994, S. 391-410, hier S. 394. 
Die Wirkung des Teufels - wunder und vndpilde - wird widersprüchlich gezeichnet. Das Unrecht, das der Teufel bewirkt, führt auch zum Wundersamen, denn das vbel kann sich ins gute wenden. Das spricht dem teuflischen Handeln eine religiöse (und narrative) Produktivität zu, die seine negative Handlungsintention übertrifft und verkehrt: Beaflor wird vor dem Vater fliehen und gerade deshalb eine legitime Ehe mit Mai eingehen können. Die Wirkungen des Teufels auf das Figurenhandeln werden in dieser Charakterisierung ebenso wie im weiteren Erzählverlauf, der auch für eine göttliche Providenz einsteht, entschärft.

\section{Schlussfolgerungen}

Die Gemeinsamkeiten dieser vier Beispiele zeichnen ein Bild des Teufels, das sich in vier Schlussfolgerungen festhalten lässt.

(1) Wenngleich der Teufel als Auslöser des Inzests auftritt, so wird dieser doch schlüssig aus der innerfamiliären Dynamik hergeleitet: aus einer familiären Zuneigung und Nähe, die aus dem Fehlen eines Elternteils entsteht und im Teilen des Betts den deutlichsten Ausdruck findet. Die knappe, aber gezielte Intervention des Teufels gibt diesem keine Präsenz als Figur, die unabhängig von den Figuren und ihrem Begehren handelt. Er verschwindet mit dem Ende der Inzesthandlung auch aus der Erzählung. Der Teufel erscheint damit als Personifizierung des Gefahrenpotentials, das in der Personenkonstellation angelegt ist.

(2) Bevor der Inzest vollzogen wird, wird eine räumliche und emotionale Nähe innerhalb der Familie etabliert. Der Teufel sieht das innerfamiliäre Liebesverhältnis und nimmt Einfluss darauf, indem er zu einer geschlechtlichen Handlung anstiftet, wobei die Figuren oft an der Grenze von kindlicher Unschuld und sexueller Reife verortet sind. Der Inzest wird damit als Einfall sexuellen Begehrens in die familiäre Nähe inszeniert. Der Teufel legt dort einen kategorialen Wechsel nahe, wo die Handlung in unguter Weise naheliegend erscheinen könnte.

(3) Denn Inzest ist wortwörtlich naheliegend. Sohn, Tochter und Schwester sind nicht nur emotional die unmittelbar nächsten, sondern auch standesmäßig und räumlich. Diese Situation familiärer Nähe bestätigt auch die kulturgeschichtliche These von FREUD und LÉVI-STRAUSS, dass nicht der Inzest, sondern vielmehr das Inzesttabu der Begründung bedürfe - dass also die Tabuisierung die entscheidende Kulturleistung sei, die gesellschaftli- 
chen Austausch antreibe. ${ }^{25}$ Gerade weil Inzest als so naheliegend erscheint, bedarf es des Teufels zur eindeutigen Markierung der Illegitimität. Der Teufel ist damit auch ein erzählerisches Mittel der Vereindeutigung.

(4) Am entscheidendsten für das narrative Potential des Teufels ist, dass er in seinem Auftreten den Figuren so nah ist, was beim Inzest besonders deutlich wird. Der Teufel verkörpert kein äußeres Böses, sondern wird vom ungeziemenden Begehren der Figuren hervorgerufen. Der Teufel ist also letztlich eine Personifizierung des Innersten, des Eigenen. Der Teufel ist nur vermeintlich ein Fremder.

25 Vgl. Eming, Jutta: Zur Theorie des Inzests. In: Genderdiskurse und Körperbilder im Mittelalter. Eine Bilanzierung nach Butler und Laqueur. Hrsg. von Ingrid BENNEWITz/Ingrid KaSTEN, Münster, Hamburg, London 2002 (Bamberger Studien zum Mittelalter 1), S. 29-48. Vgl. FreUd, Sigmund: Gesammelte Werke. Band 9: Totem und Tabu [London 1940], Frankfurt a. M. ${ }^{7} 1986$. 
